\title{
Politique
}

Politique

\section{La science politique et les sciences humaines au collégial}

\section{André Lamoureux}

Numéro 21, hiver 1992

URI : https://id.erudit.org/iderudit/040715ar

DOI : https://doi.org/10.7202/040715ar

Aller au sommaire du numéro

Éditeur(s)

Société québécoise de science politique

ISSN

0711-608X (imprimé)

1918-6584 (numérique)

Découvrir la revue

Citer cet article

Lamoureux, A. (1992). La science politique et les sciences humaines au collégial. Politique, (21), 127-146. https://doi.org/10.7202/040715ar

\section{Résumé de l'article}

Dans cet article, l'auteur tente de situer la place et le rôle de la science politique dans le programme de sciences humaines offert par les cégeps au Québec. Après avoir mis en évidence les lacunes de la formation collégiale dispensée jusqu'à maintenant dans ce domaine, après avoir souligné plus particulièrement l'éparpillement des enseignements dispensés en science politique, l'auteur essaie de mesurer l'apport que représentent le nouveau programme de sciences humaines au collégial et la nouvelle formation qui y est offerte en science politique.
Ce document est protégé par la loi sur le droit d'auteur. L'utilisation des services d'Érudit (y compris la reproduction) est assujettie à sa politique d'utilisation que vous pouvez consulter en ligne.

https://apropos.erudit.org/fr/usagers/politique-dutilisation/ 


\section{LA SCIENCE POLITIQUE ET LES SCIENCES HUMAINES AU COLLÉGIAL}

André Lamoureux

Cégep André-Laurendeau

Dans cet article, l'auteur tente de situer la place et le rôle de la science politique dans le programme de sciences humaines offert par les cégeps au Québec. Après avoir mis en évidence les lacunes de la formation collégiale dispensée jusqu'à maintenant dans ce domaine, après avoir souligné plus particulièrement l'éparpillement des enseignements dispensés en science politique, l'auteur essaie de mesurer l'apport que représentent le nouveau programme de sciences humaines au collégial et la nouvelle formation qui $y$ est offerte en science politique.

En 1990, après plusieurs années d'attente du milieu de l'enseignement collégial, le ministre de l'Enseignement supérieur et de la Science du Québec a enfin décidé d'autoriser l'entrée en vigueur d'un nouveau programme de sciences humaines dans le réseau collégial québécois. La science politique, comme toutes les autres disciplines de ce programme, a donc vu son contenu et son roble totalement redéfinis. Depuis la création des collèges d'enseignement général et professionnel (cégeps) en 1967', la science politique fait partie du programme collégial québécois en sciences humaines. Elle a d'abord fait figure de "nouvelle» discipline, comparativement à l'histoire ou à la géographie, par exemple. Comme pour bien d'autres disciplines, dont la sociologie et la science économique, l'enseignement de la science politique a dû faire son chemin à tâtons, sans pouvoir s'appuyer sur une tradition ou sur une expérience d'enseignement propre à la formation collégiale. Cet

1. Institutions d'enseignement supérieur au Québec, les cégeos représentent un niveau de formation intermediaire entre le secondaire et l'université. 
enseignement a dû aussi s'inscrire dans le cadre d'un programme de sciences humaines en gestation. D'abord considéré comme expérimental, ce programme est devenu permanent au fil du temps, malgré son manque d'unité et de cohérence.

Ainsi, après vingt longues années d'expérimentation, le programme de sciences humaines au collegial est enfin complètement refondu. Il est passé d'un assemblage de cours plus ou moins disparates à un ensemble plus homogène. Le nouveau programme s'appuie désormais sur des objectifs définis, sur un tronc commun de cours pour tous les colleges et sur une séquence de cours qui a grandement gagné en cohérence.

Dans quelle mesure peut-on observer un changement de cap dans la conception de la formation en sciences humaines au collégial? Quels sont le sens et la teneur de ce nouveau programme mis sur pied en 1991-1992? Quel est l'essentiel de la formation recherchée par l'ordre collégial en sciences humaines, et quelle place $y$ occupe la science politique? Quels sont les cours offerts en science politique, et quel apprentissage de base cherche-t-on à développer chez les étudiants? Voilà les principales questions auxquelles le présent texte tente de répondre. Favoriser une meilleure compréhension de ces changements importants, c'est certainement déjà permettre un meilleur arrimage entre la formation en sciences humaines amorcée au collégial et les divers champs de spécialisation universitaires, dont celui de la science politique.

\section{Les lacunes de l'ancien programme}

L'ancien programme de sciences humaines au collégial était fragmenté et manquait de fil conducteur. La science politique, qui en faisait partie, subissait elle aussi cette situation de morcellement.

Élaboré dans les conditions historiques, culturelles et politiques propres au Québec de la fin des années 60 , le programme de sciences humaines a été édifié selon le modèle d'organisation qui prévalait à la fondation des cégeps. Modèle qui laissait une grande latitude à l'étudiant et une grande 
liberté de choix; modèle qui donnait lieu à de grandes différences entre les collèges. II prévoyait que l'étudiant, indépendamment de son programme d'études, s'inscrive a douze cours obligatoires répartis également entre le français, la philosophie et l'éducation physique, à douze cours de concentration et à quatre cours complémentaires. En ce qui concerne la concentration en sciences humaines, aucune autre balise commune a l'ensemble du réseau n'était fixée. II appartenait à chaque collège d'organiser à sa manière ses grilles de cours selon les profils d'études qu'il décidait d'établir. L'étudiant devait cependant choisir ses cours de concentration parmi quelques disciplines reconnues dans le programme de sciences humaines, selon les grilles de cours particulières établies par son collège. II avait le choix entre quatorze matières au total : administration, anthropologie, civilisation, économie, géographie, histoire, informatique, lettres, mathématiques, philosophie, psychologie, sciences religieuses, science politique et sociologie. On constate que certaines étaient étrangères aux sciences humaines. Ces disciplines, ou quelque-unes d'entre elles, pouvaient être offertes dans un collège et pas dans un autre. Tout était fonction du choix de l'institution. Par ailleurs, l'étudiant ne pouvait choisir plus de quatre disciplines de sciences humaines et ne devait pas dépasser un maximum de six cours dans une même discipline. Toutefois, pour faciliter l'application de cette dernière règle, certaines matières étaient considérées comme une seule et méme discipline. Il en était ainsi de l'histoire et de la civilisation, de l'anthropologie et de la sociologie ainsi que de la science politique et de l'économie. Bien que cette règle, évidemment discutable, ait été fréquemment contestée, elle a été appliquée jusqu'à la récente réforme du programme des sciences humaines.

Comme on le voit, l'ancien programme comportait certains atouts, mais il contenait aussi de multiples contradictions. Le modèle d'organisation privilégiait une liberté de choix pour les collèges dans la composition des profils d'études et la liberté de l'étudiant dans ses choix de cours, en dépit de quelques restrictions peu contraignantes. L'ancien programme de sciences humaines ne prévoyait pas l'acquisition d'un apprentissage commun dans tous les 
TABLEAU 1

\begin{tabular}{l|l}
\hline \multicolumn{2}{c}{$\begin{array}{c}\text { L'ancienne banque des cours de science politique } \\
\text { des Cahiers de l'enseignement collegial }\end{array}$} \\
\hline $385-940$ & Introduction a la vie politique \\
$385-941$ & Les systèmes politiques comparés \\
$385-942$ & Les systèmes politiques du Québec et du Canada \\
$385-943$ & Les processus politiques au Québec et au Canada \\
$385-944$ & Les idées politiques modernes \\
$385-945$ & Les régimes socialistes \\
$385-946$ & Les régimes politiques du tiers-monde \\
$385-948$ & La pensée politique des indiens du Canada \\
$385-950$ & La vie politique internationale \\
$385-951$ & Les problèmes internationaux contemporains \\
$385-952$ & Introduction à l'administration publique \\
$385-953$ & La vie politique municipale \\
\hline
\end{tabular}

Source : Cahiers de l'enseignement collégial 1987-1988

1. Programmes et cours de diplómes d'études collégiales, Québec, DGEC, 1987, p.50

collèges au plan des savoirs, des savoir-faire ou des savoirêtre. Aucune base commune en méthodologie n'était prévue. Aucune compétence transdisciplinaire pourtant si importante en sciences humaines, $n^{\prime e ́ t a i t ~ v i s e ́ e . ~ L a ~ n o t i o n ~}$ d'interdisciplinarité n'était prononcée que du bout des lèvres, par les plus aventureux. À chaque discipline correspondaient des cours élaborés en fonction des plans-cadres définis au plan provincial. La configuration du programme se résumait à une juxtaposition de différentes disciplines. Dans le cadre des grilles de cours établies par son collège, l'étudiant pouvait ainsi "magasiner" sans autre contrainte, tout en respectant la limite maximale de six cours par discipline. Le programme était bel et bien caractérisé par l'émiettement et le papillonnage ${ }^{2}$. Aucune unité ne ressortait donc d'une telle formation en sciences humaines.

La science politique, comme discipline, n'échappait pas a une telle situation d'éparpillement. Les Cahiers de

2. Voir les termes utilisés dans le rapport intitulé Les colleges du Québec: nouvelle étape. Proiet du gouvernement a l'endroit des cégeps, Québec, Ministèr e de l'Éducation, 1978, p. 40 
I'enseignement collégial énuméraient douze cours de science politique. Certains étaient vraiment des cours d'introduction; d'autres étaient relativement plus et même trop spécialisés.

Quelques-uns de ces cours de science politique pouvaient être offerts dans le programme de sciences humaines. Certains pouvaient être offerts dans la concentration de d'autres programmes particuliers ou à titre de cours complémentaires au choix de l'étudiant, comme c'est toujours le cas présentement. Quant aux cours de science politique figurant au programme des sciences humaines, le choix appartenait à chaque college.

De fait, depuis la création des cégeps, presque tous les colleges ont inscrit au programme de sciences humaines des cours de science politique sans toutefois que cette discipline ait la priorité. Pour diverses raisons qu'il est impossible de mentionner ici, la psychologie, l'histoire, la -sociologie ou l'économie ont toujours conservé une place plus importante dans le programme.

En ce qui concerne la science politique, la plupart des collèges offraient le cours d'introduction à la vie politique. La presque totalité d'entre eux proposaient également l'un ou l'autre des cours de politique québécoise et canadienne, bien qu'il ait toujours été difficile d'établir la frontière entre ces deux cours. Difficile en effet de traiter des "systèmes" sans parler des "processus" et vice versa. De plus, la majorité des collèges donnaient l'un ou l'autre des cours de politique internationale. En dépit de similarités observées d'un collège à l'autre, chaque institution était libre du choix du nombre de cours dispensés en science politique. Cette pratique a créé parfois des disparités très grandes entre les collèges. II devenait ainsi impossible de s'assurer qu'une formation de base relativement homogène était dispensée dans l'ensemble des colleges.

Tout compte fait, l'enseignement de la science politique au collégial suit ce schéma : un nombre important de cours; certains cours trop spécialisés, par exemple celui sur la pensée politique des Indiens (qui n'a à peu près jamais été donné), ou le cours sur la vie politique municipale; une grande disparité de choix de cours d'un collège à l'autre; et, conséquemment, une formation de base relativement 
disparate dans l'ensemble du réseau. La science politique, comme les autres disciplines, était donc à l'image du programme des sciences humaines auquel elle est rattachée.

\section{Le processus de réforme}

Les enseignants du programme de sciences humaines ont manifesté dès le début des années 80 , la nécessité de réformer le programme en profondeur. Les lacunes étaient évidentes. Personne ne cherchait à le nier.

Après qu'un groupe de travail de la Direction générale de l'enseignement collégial eut amorcé une réflexion ${ }^{3}$, le groupe de coordonnateurs des différentes disciplines de sciences humaines au plan provincial proposa une nouvelle conception du programme. Désormais, celui-ci devait explicitement viser la préparation adéquate à des études universitaires dans l'une ou dans I'autre des sciences humaines tout en contribuant a la formation générale personnelle des étudiants". Quant aux objectifs, le ministre les regroupait autour de quatre polles : le développement de la rigueur de la pensée, l'acquisition de concepts fondamentaux et de méthodes propres aux sciences humaines, l'acquisition de connaissances de base dans quelques disciplines des sciences humaines, et l'intégration des apprentissages. Dans le cadre de ces discussions, les coordonnateurs proposèrent un modèle d'organisation du programme fondé sur trois dimensions : l'individu, la société, le monde. Cette proposition reçut l'accord des intervenants. On révisa également la définition des disciplines de sciences humaines proprement dites. Celles-ci furent ramenées à dix : administration, anthropologie, économie, civilisations anciennes, géographie, histoire, psychologie, science politique, sciences religieuses, sociologie. Seules ces dix disciplines feraient partie du bloc ministériel dans lequel les étudiants devraient désormais choisir la majorité de leurs

3. Orientations de la concentration en sciences humaines au niveau collegial. Rapport du groupe de travail, Québec, DGEC, 1983, 45 p.

4. Ministre de l'Enseignement supérieur et de la Science, Direction générale de l'enseignement collégial, Le programme révisé de Sciences humaines du collegial. Document de consultation, mai 1989, p. 2. 
cours de concentration. En fonction des objectifs poursuivis, on a pensé inclure un cours obligatoire d'initiation pratique a la méthodologie des sciences humaines et un cours de méthodes quantitatives en sciences humaines. Dans la perspective de cette orientation en trois dimensions, il a aussi été convenu d'éviter que le programme ne s'engage dans la spécialisation. Désormais, l'étudiant de sciences humaines devrait s'initier à un minimum de quatre et à un maximum de six disciplines des sciences humaines, en s'en tenant à quatre cours dans une même discipline. Partant de là, toutes les coordinations provinciales des différentes disciplines des sciences humaines durent effectuer une révision des cours offerts dans le programme. II fut aussi convenu de réduire à huit la banque de cours de chacune des disciplines de sciences humaines, dont un bloc de quatre cours dans la catégorie des cours "ministériels" et un autre bloc de quatre cours dans la catégorie "au choix des établissements" 5 .

On peut donc dire qu'en 1988, avec l'ajout de deux cours de méthodologie obligatoires et communs à tous les collèges, on était en voie d'implanter un véritable programme. L'intervention du Conseil des colleges en 1990 a permis de faire un nouveau pas ${ }^{\circ}$. Tout en souscrivant aux propositions déjà mises de l'avant dans la refonte du programme, le Conseil suggérait l'addition de deux composantes : un tronc commun de quelques cours obligatoires et l'insertion d'une activité $d$ 'intégration des apprentissages en fin de programme. Ces deux propositions du Conseil des collèges permirent de compléter l'ossature du nouveau programme en sciences humaines dans sa version définitive.

5. Dans les cours de concentration du nouveau programme, outre les cours de méthodologie, huit cours doivent etre choisis parmi les cours «ministeriels» et quatre cours parmi les cours wau choix des tablissements*. Les disciplines reconnues dans la catégorie *au choix des établissements* sont les dix de sciences humaines ainsi que quelques autres disciplines auxiliaires : mathematiques, informatique, philosophie, anglais langue seconde et français langue seconde.

6. En mars 1990 , le Conseil des colleges faisait connattre son avis au ministre de l'Enseignement supérieur et de la Science dans le cadre d'un rapport intitule Le programme revisé de sciences humaines, $57 \mathrm{p}$. 
Le tronc commun ministériel

et l'articulation du nouveau programme

La première recommandation du Conseil des collèges proposait la mise en place d'un tronc commun de quatre cours obligatoires recoupant quatre champs élargis d'études : histoire et géographie; administration et économie; psychologie; sciences sociales (sociologie, science politique et anthropologie). Le but d'un tronc commun à tout le réseau, selon le Conseil des collèges, était de remédier à «l'éclatement de la formation», d'établir un véritable programme pour tous les collèges, de créer une unité et une convergence suffisantes, et de faire connaître aux universités les paramètres précis d'une formation de base commune offerte au collégial'. Les quatre cours proposés étaient un cours d'histoire de la civilisation occidentale, un cours d'introduction à la psychologie, un cours d'économie globale et un cours de science politique (idéologies et régimes politiques). En sciences sociales, le cours de science politique a été retenu parce qu'il permettait, selon le Conseil, d'initier les étudiants aux institutions et à la vie politique, et était "réinvestissable" dans les études ultérieures et dans la vie de citoyen, en conformité avec la finalité du nouveau programme en voie d'élaboration.

L'idée du tronc commun reçut l'assentiment du milieu collégial, mais le choix du cours de science politique ne fit pas l'unanimité. Tour à tour, les coordinations de sociologie, d'anthropologie, de géographie et d'administration revendiquèrent une place dans le tronc commun. Devant les tiraillements entre les différentes disciplines, la Direction générale de l'enseignement collégial mit de côté l'argumentation développée par le Conseil des collèges et chercha plutôt à composer avec les multiples pressions exercées. En plus des deux cours de méthodologie qui reçurent l'assentiment de tous, elle prit la décision de constituer un tronc commun composé de trois autres cours obligatoires seulement (histoire, économie, psychologie), pour un total de cinq cours. Un sixième cours devait cependant

7. Conseil des colleges, op. cit. p. 28. 
s'ajouter à la formation de base de l'étudiant. Il devait être choisi par chaque collège parmi sept cours de disciplines différentes, dont la science politique. Conséquemment, cette ouverture sur sept disciplines diverses a entraîné un débordement du champ des sciences sociales privilégié au départ par le Conseil des collèges. Le raisonnement du Conseil n'a donc été adopté qu'en partie. Par la suite, chaque collège a dú décider du quatrième cours disciplinaire complémentaire. À ce jour, il n'y a pas eu de compilations des données à propos des choix effectués pour l'ensemble des collèges du Québec. Cependant, parmi les neuf cégeps de la région de Montréal, certains ont opté pour les cours de science politique, tandis que d'autres ont choisi le cours de sociologie (Individu et société) ou le cours de géographie (La carte du mondel, lesquels faisaient partie de la liste de cours soumise par la D.G.E.C. Les cégeps Ahuntsic, AndréLaurendeau, Édouard-Montpetit et Maisonneuve offrent le cours de science politique dans le tronc commun du programme de sciences humaines ${ }^{8}$.

Finalement, la composition du tronc commun dans le nouveau programme est de cinq cours ministériels obligatoires pour tous les collèges. Un sixième cours complète le tronc commun. Il doit être choisi parmi sept cours de disciplines différentes, selon les choix effectués par chaque collège. En plus de ces six cours, l'étudiant doit suivre quatre autres cours (huit unités) parmi 36 autres cours déterminés par le ministre et répartis également parmi les dix disciplines des sciences humaines. L'étudiant doit aussi choisir quatre cours (huit unités) parmi un ensemble de cours sélectionnés par chaque établissement dans une banque d'une soixantaine de cours élaborés par les dix disciplines de sciences humaines et par celles de mathématiques, de philosophie, d'informatique, de français et d'anglais. À cette formation s'ajoutent quatre cours complémentaires choisis parmi des disciplines ne faisant pas partie de la concentration sciences humaines.

8. Voir Le nouveau programme de Sciences humaines, document produit par les cépeps de la région de Montréal, mars 1992, p. 20. 
L'intégration des apprentissages et la mise en ceuvre du nouveau programme

La deuxième proposition du Conseil des collèges était d'ajouter en fin de programme un cours d'intégration des apprentissages. Cette activité créditée, équivalant à un cours de concentration, devait permettre a l'étudiant de vérifier par lui-même le caractère intégré de l'apprentissage en sciences humaines, d'arriver "à un certain niveau d'intégration et de synthèse, et d'en faire explicitement la démonstration" ". Cette deuxième proposition du Conseil des collèges, malgré toute sa pertinence, n'a pas été appliquée à ce jour dans le nouveau programme. Même si l'objectif d'intégration des apprentissages a été maintenu par le ministre ${ }^{10}$, la proposition d'instaurer une activité supplémentaire créditée pour y parvenir n'a pas été retenue. La seule explication connue de ce refus est le cout supplémentaire que cette activité aurait ajouté à celui de l'implantation du nouveau programme. Bref, pour une question de gros sous, l'objectif d'intégration des apprentissages n'a pas connu de traduction concrète dans le programme et s'est même perdu dans les méandres de la réforme. Fait révélateur, le nouveau programme en sciences humaines, publié en 1991 par la Direction générale de l'enseignement collégial, omet même d'identifier l'intégration des apprentissages parmi les objectifs. Qu'il s'agisse d'un simple oubli ou d'un acte délibéré, il reste que le débat autour de la mise en œuvre d'une activité $d^{\prime}$ intégration des apprentissages reste entier.

Malgré tout, dans la foulée de tous ces événements et avec la pression exercée par le milieu pendant de longues années, le ministre a autorisé, en juin 1990, la mise en œuvre du nouveau programme de sciences humaines. C'était là, bel et bien, une victoire du milieu. Les mois suivants ont servi a l'organisation du programme et aux préparatifs nécessaires dans chaque collège. L'année 1991-1992 a signé l'inaugu-

9. Conseil des colleges, op. cit. p. 31.

10. Voir la lettre du ministre Claude Ryan autorisant officiellement l'entrée on vigueur du nouveau programme, publiée le 27 juin 1990. 


\section{Le contenu du nouveau programme collégial en sciences humaines}

\begin{tabular}{|c|c|}
\hline $\begin{array}{l}\text { LE TRONC COMMUN } \\
\text { MINISTÉRIEL (CINQ COURS) } \\
\text { (tronc commun obligatoire } \\
\text { dans tous les colleges) }\end{array}$ & $\begin{array}{l}\text { Initiation pratique a la } \\
\text { méthodologie des sciences } \\
\text { humaines (300-300) } \\
\text { Méthodes quantitatives en } \\
\text { sciences humaines }(360-300) \\
\text { Histoire de la civilisation } \\
\text { Hocidentale (330-910) } \\
\text { occion } \\
\text { Introduction a la psychologie } \\
\text { (350-102) } \\
\text { Economie globale (383-920) }\end{array}$ \\
\hline $\begin{array}{l}\text { UN COURS PARMI LES SEPT } \\
\text { SUIVANTS }\end{array}$ & $\begin{array}{l}\text { *a carte du monde (320-103) } \\
\text { - Ala recherche des civilisations } \\
\text { disparues (332-903) } \\
\text { - Sur la piste des Dieux } \\
\text { introduction a l'étude des } \\
\text { religions (370-111) } \\
\text { - L'espece humaine et son } \\
\text { évolution (381-900) } \\
\text { - ldéologies et régimes politiques } \\
\text { (385-941) } \\
\text { - Individu et société(387-960) } \\
\text { - L'entreprise (401-913) }\end{array}$ \\
\hline $\begin{array}{l}\text { HUIT UNITES (QUATRE } \\
\text { COURS) CHOISIES PARMI LES } \\
36 \text { AUTRES COURS } \\
\text { MINISTERIELS PREPARES PAR } \\
\text { LES DISCIPLINES SUIVANTES : } \\
\text { (selon I'eventail des cours } \\
\text { ministeriels spécifiques } \\
\text { retenus et offerts dans } \\
\text { chaque college en particulier) }\end{array}$ & $\begin{array}{l}\text { - Geographie } \\
\text { Histoire } \\
\text { * Civilisations anciennes } \\
\text { *sychologie } \\
\text { - Sciences religieuses } \\
\text { Anthropologie } \\
\text { * Economie } \\
\text { - Science politique } \\
\text { - Sociologie } \\
\text { Administration } \\
\end{array}$ \\
\hline $\begin{array}{l}\text { HUIT UNITES (QUATRE } \\
\text { COURS ') PARMI UNE LISTE } \\
\text { DUUNE SOIXANTAINE DE } \\
\text { COURS AU CHOIX DES } \\
\text { ETABLISSEMENTS, PREPARES } \\
\text { PAR LES DISCIPLINES } \\
\text { SUIVANTES : } \\
\text { " Trois cours s'il s'agit de } \\
\text { cours de mathématiques) }\end{array}$ & $\begin{array}{l}\text { Les dix disciplines des sciences } \\
\text { humaines et: mathématiques, } \\
\text { philosophie, informatique, } \\
\text { français et anglais. }\end{array}$ \\
\hline $\begin{array}{l}\text { QUATRE COURS } \\
\text { COMPLEMENTAIRES (HUIT } \\
\text { UNITES) }\end{array}$ & $\begin{array}{l}\text { - Choisis dans des disciplines hors } \\
\text { concentration }\end{array}$ \\
\hline
\end{tabular}


ration dudit programme de sciences humaines.

En dépit des compromis consentis en cours de route et malgré certains aspects inachevés, le nouveau programme donne indéniablement plus de cohérence a la formation collégiale en sciences humaines. L'existence d'un tronc commun de cours obligatoires, incluant des cours de méthodologie et d'autres cours de base rattachés à quelques disciplines, permet de considérer le tout comme un réel programme. L'ancien éparpillement est évité. Un fil conducteur, une unité et une cohésion sont apparus. C'est déjà beaucoup. Cependant, on s'interroge encore sur l'intégration des apprentissages. A ce propos, des discussions et un processus d'expérimentation sont en marche avec la possibilité d'instaurer un examen-synthèse de fin de programme, selon une nouvelle suggestion mise de l'avant par le Conseil des collèges. Mais il reste que le programme a énormément gagné en qualité. Par ailleurs, il s'agit d'un programme beaucoup plus exigeant que le précédent, ce qui contribue aussi à rehausser l'image de la formation collégiale en sciences humaines, trop souvent dévalorisée dans l'opinion publique.

La science politique sous un nouveau jour

Dans l'élaboration du nouveau programme, la science politique, comme toutes les autres disciplines des sciences humaines, a dû faire le ménage dans ses propres cours. L'ensemble des cours anciennement offerts a été remis en question par la coordination provinciale de science politique à laquelle participent des délégués de tous les collèges. Les objectifs poursuivis ont été à la fois de réduire à huit le nombre de cours de science politique offerts en sciences humaines, de les redéfinir en totalité en fonction d'une mise à jour des connaissances recherchées au collégial, de les rendre prioritaires et de suggérer un logigramme pour l'enchainement des cours ministériels.

Les membres de la coordination provinciale de science politique ont d'abord dû choisir les quatre cours ministériels de la discipline, c'est-à-dire pouvant figurer, partiellement ou entièrement, dans le bloc des cours ministériels en fonction 
des choix opérés par chaque institution. Les cours ministériels, rappelons-le, sont les cours prépondérants de chaque discipline. Dans l'articulation du programme, ils ont plus de poids et ont plus de chance d'être suivis par l'étudiant, qui doit choisir la majorité de ses cours de concentration dans cette banque. La logique suivie a été d'offrir des cours permettant à l'étudiant de s'initier à la science politique et d'acquérir les connaissances et les méthodes de base dans cette discipline. Selon cette logique, les quatre cours ministériels retenus furent les suivants ${ }^{11}$ :

* La vie politique (385-940) [un cours d'initiation a la science politique];

- Idéologies et régimes politiques (385-941);

- La politique au Canada et au Québec (385-942);

* Actualité politique internationale (385-950).

Ces quatre cours du bloc ministériel répondent à quatre préoccupations : initier les étudiants à la vie politique, à la discipline et à ses méthodes; les sensibiliser aux grandes idéologies et aux différents régimes politiques; enfin, les familiariser avec deux grands champs d'analyse de la science politique que sont la dynamique politique au Québec et au Canada et la vie politique internationale. Ce bloc permet l'acquisition de l'a.b.c. de la science politique. Toutefois, tous les collèges n'offrent évidemment pas ces quatre cours en bloc. Mais, indépendamment des choix institutionnels, il importe de retenir que chacun de ces cours a été conçu de manière à permettre une initiation aux notions de base de la discipline.

D'autre part, compte tenu de l'existence d'un tronc commun de cours obligatoires dans le programme et de la possibilité, comme nous l'avons déjà expliqué, d'y inscrire un cours de science politique, le cours /déologies et régimes politiques a pris une importance indéniable dans plusieurs

11. Voir la description détaillée des cours annexée à la fin de l'article. 
colleges. Ce cours sert souvent à la fois d'introduction au domaine de la science politique et de cours d'initiation a la vie politique.

Les quatre autres cours de science politique, réunis dans la catégorie "au choix des établissements", sont les suivants :

* Les idées politiques modernes (385-944);

- Tiers-monde et politique internationale (385-946);

- Administration publique et parapublique (385-952);

- Groupes de pression et opinion publique (385955).

Dans le nouveau programme, ces quatre derniers cours sont plus spécialisés. Ils ont aussi moins de prépondérance que les cours «ministériels». Cependant, les collèges peuvent inscrire l'un ou l'autre de ces cours au programme de sciences humaines.

\section{Conclusion}

La science politique, au collégial, a bénéficié de la réforme des sciences humaines sur un double terrain : d'une part, elle est mieux intégrée au programme, compris comme un tout. Elle n'est plus simplement une discipline juxtaposée aux autres, et quelques collèges ont opté pour le cours de science politique comme complément de leur tronc commun de formation en sciences humaines. D'autre part, la mission proprement collégiale de la science politique a été mieux définie. A ce propos, les cours du bloc ministériel correspondent assez fidèlement à l'un des grands objectifs du programme, qui vise l'acquisition des concepts fondamentaux et des connaissances de base dans la discipline plutôt que la spécialisation trop rapide.

A la fondation des cégeps, la nature des cours de science politique choisis dans les Cahiers de l'enseignement collégial, leur nombre, leurs objectifs et leurs contenus n'ont 
pas fait l'objet d'une large consultation et d'une concertation dans le réseau. La spécificité de ces cours par rapport au niveau universitaire n'était pas non plus toujours évidente. $A$ vrai dire, le premier véritable mouvement de concertation et de débat sur le sujet a l'intérieur de la discipline s'est matérialisé dans l'actuel processus de réforme du programme des sciences humaines, qui prend forme aujourd'hui. II faut dire que ce cheminement a exigé plusieurs années avant d'aboutir. Mais cet échange a permis entres autres choses de définir quatre cours ministériels qui se veulent l'a.b.c. de la science politique au collégial. Malgré certaines variantes d'une institution a l'autre, les cours de science politique seront tirés pour la plupart de ce bloc de quatre cours, ce qui va renforcer l'harmonisation de la formation en science politique dans le réseau collégial. Cette plus grande harmonisation favorisera à son tour une préparation plus adéquate de tous les étudiants qui poursuivront des études universitaires, quel que soit le collège qui les aura formés.

La réforme du programme des sciences humaines qui voit le jour au collégial n'est certainement pas parfaite. Certains aspects restent inachevés ou ont été mis de côté pour des motifs strictement financiers. Certaines autres carences subsistent. Mais l'existence d'un tronc commun de formation, si limité soit-il, marque un indéniable progrès par rapport a la formation éclatée et éparpillée qui était donnée auparavant. Dans cet effort de réforme, le rôle et la spécificité de la science politique ont été mieux définis. 
Les quatre cours ministériels en science politique ${ }^{12}$

385-940-91

LA VIE POLITIQUE

\section{BUTS}

Fournir une vue d'ensemble des phénomènes d'État et de gouvernement à l'aide de l'analyse scientifique. Dépasser les préjugés et les stéréotypes en vue d'acquérir un sens critique face aux enjeux politiques. Développer un intérêt et prendre conscience des questions politiques, afin de devenir une citoyenne, un citoyen avertis.

\section{OBJECTIFS}

Au terme de ce cours, l'élève sera capable : 1 . De distinguer les objets et méthodes de la science politique. 2. De situer les concepts de base qui permettent de connaitre, comprendre, expliquer et prévoir les phénomènes politiques. 3. De définir les principaux éléments du vocabulaire et du discours politiques. 4. De distinguer les nombreuses formes du pouvoir, de l'organisation et du conflit politiques. 5. D'utiliser des approches qui favorisent l'analyse objective et rigoureuse des institutions et des processus politiques.

\section{CONTENU}

Objets de la science politique : le pouvoir, les structures, les forces, les idées et les aspirations. La méthode historique, la méthode comparative, l'analyse de contenu. Concepts d'État, de pouvoir et de légitimité. De l'antagonisme à l'intégration : notions de conflit et de violence, le rôle du gouvernement dans la résolution des conflits. Les enjeux politiques, les facteurs socio-économiques et idéologiques. Institutions et régimes politiques au $X X^{\bullet}$ siècle. Les applications à l'analyse 
du discours politique par l'étude de crises ou de négociations. Étude de l'influence sur l'entourage immédiat des élèves, des élections, de leur participation et de leur engagement.

385-941-91

IDÉOLOGIES ET RÉGIMES POLITIQUES

BUTS

Se familiariser avec les principales idéologies politiques. Réfléchir de façon critique sur les courants politiques et leurs rapports avec les régimes et institutions politiques. S'ouvrir à d'autres idéologies et régimes politiques.

\section{OBJECTIFS}

Au terme de ce cours, l'élève sera capable : 1. D'appliquer la méthode comparative par l'étude des principales idéologies politiques et régimes politiques. 2. De distinguer les systèmes de valeurs des principales idéologies. 3. De définir les caractéristiques propres aux principaux régimes politiques actuels. 4. De connaître les liens entre les idéologies et leur expression dans des institutions politiques spécifiques. 5. D'expliquer des liens entre les nouvelles valeurs, courants politiques et leur traduction éventuelle dans des régimes politiques.

\section{CONTENU}

1. La méthode comparative, idéologies politiques actuelles et régimes politiques correspondants. 2 . Variables comparatives à privilégier : fondements socio-économiques et culturels, rôle de l'État, institutions, conquête du pouvoir. 3. Partis politiques et processus électoral. 4. Dynamique de la vie politique à travers la participation ou la non-participation de citoyens. 5. Autoritarisme politique ou politico-religieux, régime à parti unique, non socialiste ou militaire. 6. Libéralisme, démocratie fondée sur le concept de liberté. 
7. Socialisme, démocratie fondée sur le concept d'égalité. 8. Importance des valeurs traduites dans les courants politiques dominants et exerçant une influence formelle ou non sur les régimes politiques : écologisme, pacifisme, théologie de la libération, féminisme et internationalisme.

385-942-91

LA POLITIQUE AU CANADA ET AU QUÉBEC

BUTS

1. Fournir une vue d'ensemble de l'analyse scientifique de la politique au Canada et au Québec. 2. Apprendre à identifier les interactions affectant l'exercice du pouvoir et influençant la prise de décision politique. 3. Développer un intérêt envers l'environnement politique ambiant.

\section{OBJECTIFS}

Au terme de ce cours, l'élève sera capable : 1 . De reconnaître la spécificité du système politique canadien et québécois a travers les facteurs historiques, géographiques, socioéconomiques et culturels qui l'ont modelé. 2. De distinguer les facteurs qui fondent la particularité de la société politique québécoise dans l'ensemble canadien. 3. D'identifier la dynamique propre du fonctionnement du régime fédéral. 4. De décrire les principales institutions politiques canadiennes et québécoises. 5. De connaître les processus menant à la prise de décision politique. 6 . De critiquer l'actualité politique à travers l'étude de différents documents juridiques et politiques.

\section{CONTENU}

1. Les grandes caractéristiques de la société politique canadienne : environnement et particularismes. 2. Le fédéralisme et les relations fédérales/provinciales. 3. La question constitutionnelle et son évolution. 4. Le 
parlementarisme au Canada et au Québec. 5. Les institutions du régime politique : la Couronne, le gouvernement, le Parlement, l'administration, les tribunaux. 6. Le processus de décision et les intervenants : partis, groupes de pression, opinion publique, les sondages, le système électoral. 7. Les éléments constitutionnels : textes, interprétations et conventions.

385-950-91

ACTUALITÉ POLITIQUE INTERNATIONALE

BUTS

Prendre conscience de la complexité des relations de pouvoir et de puissance qui existent dans le monde actuel. Évaluer les influences et les rapports de force qui déterminent la conduite des États et des autres acteurs internationaux. Développer l'intérêt et l'ouverture à la vie politique internationale.

\section{OBJECTIFS}

Au terme de ce cours, l'élève sera capable : 1. D'utiliser des approches qui permettent la comparaison des enjeux, des acteurs, des ressources et des stratégies sur la scène internationale. 2. De décrire les fondements de la société internationale. 3. De connaître certains phénomènes conflictuels de la politique internationale. 4. D'identifier les principaux problemes politiques internationaux actuels. 5. D'expliquer avec pertinence des grands problèmes internationaux.

\section{CONTENU}

1. Les relations internationales, objet et champ d'étude. 2. Concepts et méthodes d'analyse, approches et écoles. 3. Les facteurs des relations internationales et les réalités géopolitiques et stratégiques : intérêts économiques, fondements institutionnels et caractères socioculturels et 
idéologiques. 4. Les acteurs : les États, les organisations internationales, régionales, et les forces transnationales. 5. Notions de pouvoir, de conflit et de paix. 6. Les quatre $D$ : désarmement, décolonisation, développement, démocratisation. 7. Les enjeux d'une nouvelle configuration mondiale : multilatéralité et nouvelles alliances. 8. Étude de certains problèmes internationaux actuels. 9. Le Canada et le Québec sur la scène internationale. 